Research Paper

\title{
S100A4-MYH9 Axis Promote Migration and Invasion of Gastric Cancer Cells by Inducing TGF- $\beta$-Mediated Epithelial-Mesenchymal Transition
}

\author{
Fengping $\mathrm{Li}^{*}$, Jiaolong Shi*, Zhijun $\mathrm{Xu}^{*}$, Xingxing Yao, Tingyu Mou, Jiang Yu, Hao Liu ${ }^{\bowtie}$, Guoxin Li ${ }^{\bowtie}$ \\ Department of General Surgery, Nanfang Hospital, Southern Medical University, Guangdong Provincial Engineering Technology Research Center of Minimally \\ Invasive Surgery, Guangzhou, China \\ * These authors contributed equally to this work and should be considered co-first authors. \\ $\bowtie$ Corresponding authors: Guoxin Li, email: nfykdxpwk@163.com; Hao Liu, email: liuhaofbi@163.com \\ (C) Ivyspring International Publisher. This is an open access article distributed under the terms of the Creative Commons Attribution (CC BY-NC) license \\ (https://creativecommons.org/licenses/by-nc/4.0/). See http://ivyspring.com/terms for full terms and conditions.
}

Received: 2018.02.09; Accepted: 2018.07.17; Published: 2018.10.05

\begin{abstract}
Driver genes conducing to peritoneal metastasis in advanced gastric cancer remain to be clarified. S100A4 is suggested to evolve in metastasis of gastrointestinal cancer, we aim to explore the role of S100A4 plays in metastasis of advanced gastric cancer and the potential mechanism. Transfection of siRNA or cDNA was applied to alter the expression of protein S100A4 and MYH9, investigation of the expression of epithelial and mesenchymal transition (EMT) associated markers was followed. Cell migration assay was used to screen the alteration of migration ability regulated by S100A4 and MYH9. IHC analysis for tissue sample microarray was performed to reveal their relationship with clinical pathological parameters and potential capacity of predicting survival. Consistent overexpression of S100A4 and MYH9 were found in peritoneal metastasis and primary site compared with adjacent normal tissue. Low expression of S100A4 led to increased epithelial markers as wells as decline of mesenchymal makers, while overexpression of S100A4 led to inverse impact. S100A4 expression was closely correlated with increased migration ability and EMT process induced by TGF- $\beta$ stimulation. Interference of S100A4 led to downregulation of MYH9 and inactivation of Smad pathway through participating in EMT process, which could be reversed by overexpression of MYH9. Moreover, co-expression of S100A4 and MYH9 was identified in tissue microarray and confirmed by immunofluorescence assay. In conclusion, overexpression of S100A4 and downstream molecular MYH9 in advanced gastric cancer predicted poor prognosis; oncogene S100A4 facilitate EMT process induced by TGF- $\beta$ stimulation, suggesting a potential target in management of peritoneal metastasis of gastric cancer.
\end{abstract}

Key words: S100A4, peritoneal metastasis, gastric cancer, EMT, MYH9

\section{Introduction}

Gastric cancer is one of the most common malignant tumor and the third leading cause of cancer death worldwide[1-3]. Approximately 951600 new gastric cancer cases and 723100 deaths occurred in 2012 in the world, while many of patients are diagnosed in advanced stage with a high risk of metastasis and recurrence in China[4, 5].Peritoneal carcinoma is the most common pattern of metastasis and recurrence accounting for $20.0-53.5 \%$ of recurrence after radical resection for gastric cancer[6-8] .The median survival of patients with peritoneal metastasis is less than 6 months, owing to the rapid progression of metastasis and development of resistance to kinds of therapy[9-13]. However, effective treatments for peritoneal carcinomatosis are still undefined.

Metastasis of tumor is a complex process involving multiple pathogenic steps and many metastasis-related proteins have been identified to be involved. However, we have not yet succeeded in deciphering the molecular mechanism associated with peritoneal metastasis in gastric cancer. Recent 
advancement has illustrated that the epithelialmesenchymal transition (EMT) is a key mechanism in the invasiveness and metastatic initiation in most cases of carcinoma[14].

During EMT process, cells develop with the increased mobility and initiate migratory characteristics, contributing to a multiple biochemical change in epithelial cells and thereby enabling it to assume a mesenchymal cell phenotype[15]. Cancer cells acquire aggressive properties and intrude into the surrounding stroma through the process, accompanied with the reduced apoptosis and resistance to some exogenous agents[16]. It's urgent for us to explore the driver molecules involved in EMT process, which makes access to exploitation of specific drugs targeting the peritoneal metastasis potential of gastric cancer.

S100A4 is one of the numerous specific metastasis-related proteins, which was synthesized as a 9,000-molecular weight acidic polypeptide (p9ka/S100A4) by the elongated myoepithelial-like cells in a rat mammary tumor cell line culture[17, 18] .The human S100A4 gene is localized in chromosome 1q21 and consists of four exons that code a protein with 101 amino acid residues. S100A4 is a member of the $\mathrm{S} 100$ calcium binding protein family and is also known as metastasin p-EL-98, 18A2, 42A, p9Ka, CAPL, calvasculin, and fibroblast-specific protein. S100A4 was identified by Ebradlize and colleagues, and shown to be associated with tumor metastasis for the first time[19] .Our previous study has confirmed that S100A4 played an important role in TGF- $\beta$-induced EMT in colorectal cancer[20]. Those all suggest potential ability of S100A4 in the invasion and metastasis of human malignant tumors.

To figure out role of S100A4 plays in the peritoneal metastasis of gastric cancer, we investigate the involvement of S100A4 in EMT process of gastric cancer by demonstrating its relation with EMT-associating markers in tissue samples of human gastric cancer. We also determined its role on TGF$\beta$-induced EMT and cell invasion in gastric cancer cell lines by in-vitro experiments of lost-function and gain-function. We aimed to gain deeper insight into molecular mechanisms of peritoneal metastasis in gastric cancer and shed new light on target therapy for patients with advanced gastric cancer and peritoneal metastasis.

\section{Materials and Methods}

\section{Cell lines and tumor tissue samples}

The gastric cancer cell lines MKN45, MGC803, SGC7901 were obtained from the Cell Bank of the Chinese Academy of Science (Shanghai, China) and maintained as previously described[21]. These cells were cultured with RPMI Medium 1640(Thermo Fisher, Life Technologies Corporation; Grand Island, NY) supplemented with $10 \%$ fetal bovine serum (FBS) at a humidity of $5 \% \mathrm{CO}_{2}$ at $37^{\circ} \mathrm{C}$. As for TGF- $\beta$ treatment, the cells were stimulated with human recombinant TGF- $\beta 1$ (Peprotech), which was diluted with serum-free medium containing BSA for 24 or 48 hours. Fresh primary gastric cancer specimens and paired noncancerous gastric tissue specimens were provided by the Department of General Surgery in Nanfang Hospital of Southern Medical University in Guangdong, China.

\section{Plasmids and transfection}

A plasmid containing S100A4 cDNA was purchased from OBIO Biotechnology Company. S100A4-specific siRNAs were synthesized as previously described[20].Exponential growth phase cells were planted in 6-well plates at a density of $0.5 \times 10^{5}$ cells $/ \mathrm{mL}$ and starved for 24 hours, then $1 \mathrm{mg}$ of siRNA or $4 \mathrm{ug}$ cDNA in reduced serum medium were transfected(OPTI-MEM I; Invitrogen) according to the manufacturer's protocol.

\section{Western blotting}

Proteins were extracted with RIPA lysate and separated(20ug) on SDS-PAGE gels, then the following antibodies were incubated overnight with gently shaking at $4^{\circ} \mathrm{C}$ : rabbit antibody to S100A4 (1:500; Abcam), E-Cadherin, $\beta$-Catenin, N-Cadherin (1:1000; Proteintech), p-AKT (ser473), AKT, p-c-raf259, p-P44/42, p-Smad2/3, Smad2/3 (1:1000; CST); and mouse antibodies to MYH9 (1:1000; Abcam), GAPDH, Vimentin (1:1000; Proteintech).

\section{Immunohistochemistry}

As previously described, IHC was performed to investigate the expression of proteins in human gastric cancer tissue[22]. The sections were incubated overnight with primary antibodies against S100A4, MYH9 (1:100), E-Cadherin, $\beta$-Catenin, Vimentin (1:50) at $4^{\circ} \mathrm{C}$. Mayer's hematoxylin was applied to nuclear counter staining. In this study, our slides were reviewed by two or three blindfolded pathologists. To evaluate the expression levels of protein, the intensity of staining of cancer cells was scored as follows: 0 (no staining), 1 (weak staining, light yellow), 2 (moderate staining, yellow brown), and 3 (strong staining, brown). The ultimate immunohistochemistry score for individual case were listed as Supplementary table 1. An intensity score of $\geq 2$ was considered as high expression (or overexpression), whereas the rest of the intensity score was regarded as low expression. The discrepancies $(<5 \%)$ were resolved by simultaneous 
reevaluation. The significance of correlation was determined using the Pearson $\chi^{2}$ test.

\section{Cell Migration assay}

For migration assay, $10^{5}$ cells mixed with $0.2 \mathrm{ml}$ serum-free culture medium were filled in the upper chamber of transwell plate (Corning) as reported[21]. In the lower chamber, $0.6 \mathrm{ml}$ medium with $10 \%$ of FBS were abounded to attract the cells of the upper chamber to squeeze into the holes of the membrane. After incubation for 24 hours at $37^{\circ} \mathrm{C}$, cells penetrated the membrane were fixed with methanol solution for 15 minutes, Giemsa staining was used to mark the migrated cells, the number of cells in 4 randomly selected microscope fields were counted.

\section{KM plotter analysis}

Kaplan-Meier plotter (http://www.kmplot. com), an online tool that incorporates public microarray data from GEO and TCGA database, was used to analyze the transcription levels and prognosis value of S100A4 in 876 patients with gastric cancer[23]. Patient samples were split into two groups according to the median expression (high vs. low expression) and assessed by a Kaplan-Meier survival plot, with the hazard ratio (HR) with 95\% confidence intervals $(\mathrm{CI})$ and log rank $\mathrm{p}$ value.

\section{Results}

\section{S100A4 is significantly correlated with expression of EMT markers in gastric cancer}

Immunoblotting analysis was performed to detect the expression of S100A4 in gastric cancer tissues. We discovered relatively high expression level of S100A4 in 62.5\% (5/8) gastric cancer tissue compared to adjacent normal sample. Interestingly, the inversely correlative expression of E-Cadherin with S100A4 was observed in $50 \%$ (4/8) gastric cancer tissues (Figure 1A and Supplementary table 1). In this study, IHC detection for the expression of S100A4, E-Cadherin, $\beta$-catenin and Vimentin in additional 20 paired of matched tissues confirmedly revealed the correlation between expression of S100A4 and E-Cadherin, $\beta$-catenin, Vimentin. S100A4 expression was closely positively correlated with Vimentin, while inversely correlated with E-Cadherin and $\beta$-catenin expression. Furthermore, we noticed that S100A4 was significantly upregulated in the invasive margin that surrounded the micro invasive foci of gastric cancer (Figure 1B).

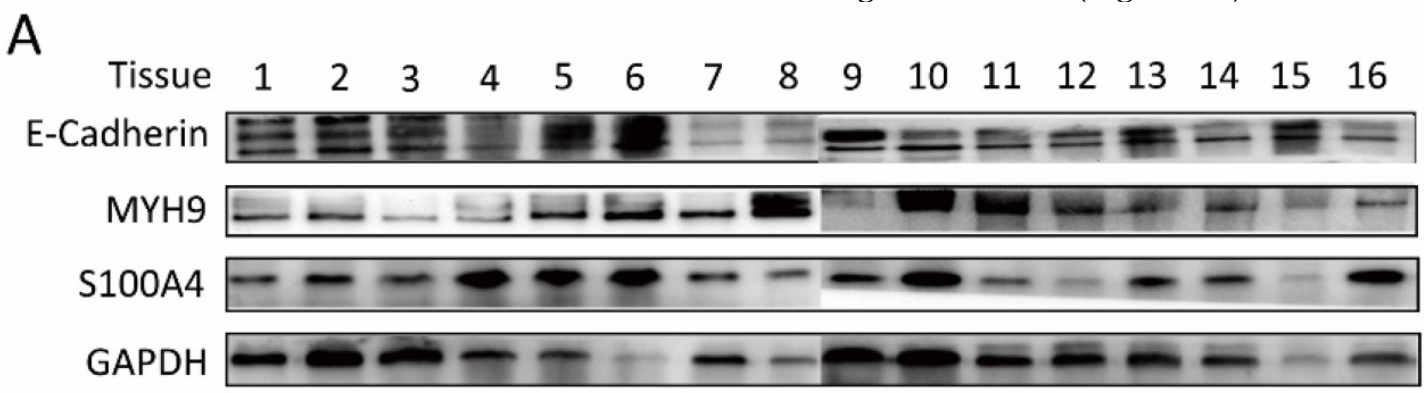

B
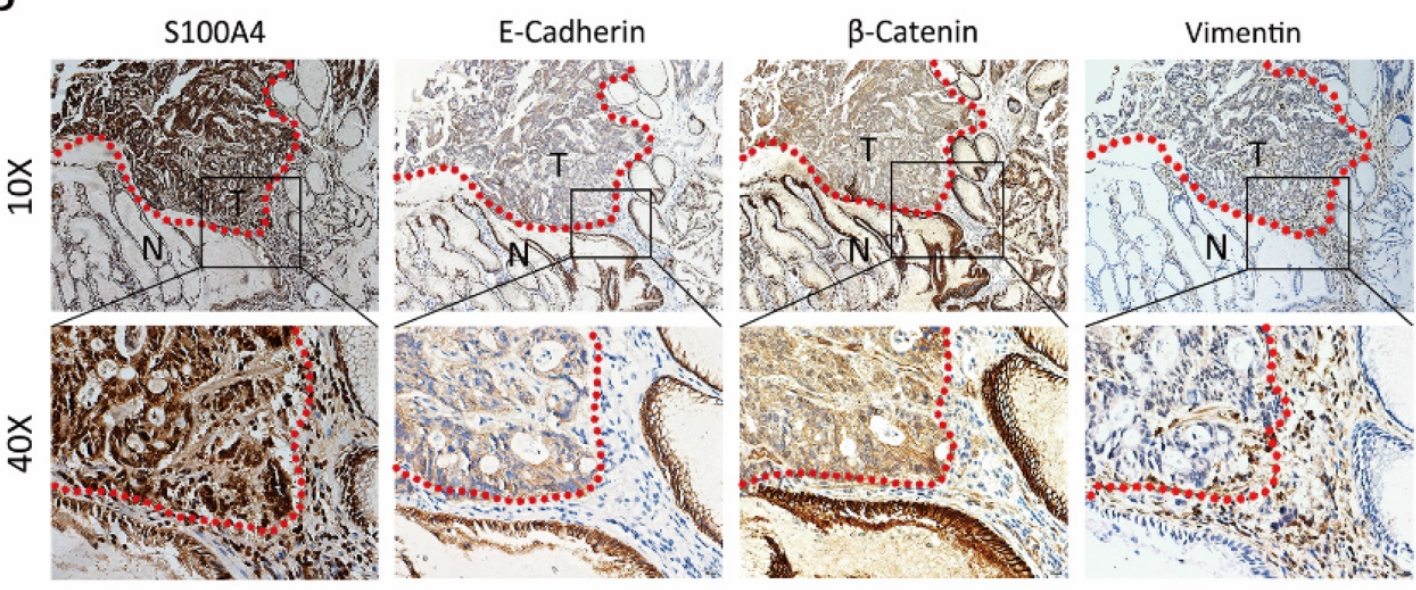

Figure 1. S100A4 was correlated with expression of EMT markers in gastric cancer tissue. A, immunoblotting analysis is performed to detect the expression of S100A4, MYH9, and E-Cadherin in gastric cancer tissues. B. S100A4 was up-regulated in the invasive margin and surrounded the micro invasive foci of gastric cancer and relative to the level of E-Cadherin, $\beta$-Catenin and Vimentin. 


\section{S100A4 enhanced cell invasiveness through induction of EMT}

Loss of function assays in vitro were employed to confirm above results derived from analysis for clinical samples. After transfection of S100A4 targeted siRNA, increased expression of epithelial markers (E-Cadherin and $\beta$ - catenin) and decreased expression of mesenchymal markers (Vimentin and N-Cadherin) were observed in MKN45 and MGC803 cells (Figure $2 \mathrm{~A}$ and $\mathrm{C}$ ), indicting low expression of S100A4 suppressed the transition of epithelial to mesenchymal. Transwell assay suggested that after being knock down of S100A4, invasive ability of MKN45 and MGC803 cells were obviously lower compared to control group (Figure 2B).

To investigate the influences of S100A4 on the expression of EMT associated markers in gastric cancer and peritoneal metastasis, we detected and analyzed the correlation between S100A4 and EMT markers by using IHC assay. S100A4 was overexpressed in gastric cancer and peritoneal foci along with upregulated mesenchymal markers Vimentin. In contrast, epithelial marker E-Cadherin was downregulated in gastric cancer and matched peritoneal foci tissues but overexpressed in normal gastric tissues (Figure 2D).

\section{S100A4 regulated Smad pathway to induce EMT progress}

To explore molecular mechanism underlying S100A4-mediated EMT, we carried out Western blot analysis for phosphorylation status of proteins in EMT signaling. Overexpression of S100A4 significantly induced phosphorylation of Smad2, and AKT in SGC7901 cells, indicating its role of a key mediator of EMT through Smad and AKT signaling pathway (Figure 3A, B). To further elucidate the relationship between S100A4 and TGF- $\beta$ signaling in gastric cancer, we analyzed the effect of stimulation of recombinant TGF- $\beta$ on S100A4 expressions in MKN45 and MGC803 cells. We identified that TGF- $\beta$ stimulation lead to evident increase of S100A4 expression level, upregulated epithelial markers and down regulated mesenchymal markers which indicated EMT process (Figure 3C). After being treated with TGF- $\beta$ for 48 hours, MKN45 and MGC803 cells showed characteristic appearance of cobblestone-like phenotype (Figure 3D) and morphologic changes of EMT, we also observed time-dependent increases in migration capacity of tumor cells (Figure 3E).

\section{MYH9 co-expressed with S100A4 in gastric cancer}

Through searching for String.com database we found that MYH9 was one of candidates of S100A4 modulated proteins (Figure 4A). To determine their association in cell lines, we interfered expression of S100A4 in MGC803 cells by transfection of siRNA. Q-PCR analysis suggested that the relative mRNA level of MYH9 was reduced which shown the same tendency with S100A4 (Figure 4B). In protein level, the expression of MYH9 was always in the wake of expression change of S100A4 result from knockdown of S100A4 or arise from transfection of S100A4 plasmid in MKN45 cells and MGC803 cells (Figure $4 C, D)$. We visualized co-expression of the two proteins by immunofluorescence assay of MKN45 and MGC803 cells (Figure 4E). The mutual interaction between S100A4 and MYH9 was confirmed by Co-immunoprecipitation assay (Figure $4 \mathrm{~F}$ ).

To illustrate correlation between expression of S100A4 and MYH9 in a clinical setting, we selected a piece of sample microarray covered with 92 stomach cancer tissues for IHC detection. We discovered that expression level of MYH9 was frequently higher in samples with high-scored S100A4 expression (Supplementary Table 1) compared to that with low-scored S100A4. MYH9 expression was obviously correlated with S100A4 expression $\left(R^{2}=0.7160, p<0.0001\right.$; Figure $4 \mathrm{G}, \mathrm{H})$. Prognosis significance of S100A4 mRNA expression in GC patients was investigated by searching in Kaplan-Meier plotter database. We identified that S100A4 was not significantly correlated to overall survival of GC patients but high S100A4 expression remained a highly significant indicator of poor prognosis in diffuse type or stage IV GC patients, suggesting that S100A4 might correlate with more invasive behavior and treatment resistance in gastric cancer (Figure 4 I, J, K).

\section{S100A4 and MYH9 are critical for TGF- $\beta$ mediated EMT process}

We investigated the roles of S100A4 coupled with MYH9 in the EMT process, which was regulated by TGF- $\beta /$ Smad pathway. TGF- $\beta$ stimulation promoted the expression of MYH9 in MKN45 cells and MGC803 cells in a time-dependent manner as S100A4 has assumed (Figure 5A). TGF- $\beta$ stimulation facilitated migration capacity of MKN45 cells MGC803 cells while knock of S100A4 or MYH9 impaired this impact (Figure 5B, C). Besides, transfection of S100A4 or MYH9 specific siRNA could partially suppress progression of EMT which was manifested as mesenchymal makers and reduced expressions of epithelial makers. Western blot analysis showed that interference of S100A4 or MYH9 
was able to suppress the phosphorylation of AKT, and Smad2. Either interference of S100A4 or MYH9 obviously give rise to inactivation of Smad pathway which was activated by TGF- $\beta$ stimulation (Figure 5D). We consider that both of S100A4 and MYH9 are required for TGF- $\beta$ mediated EMT process.

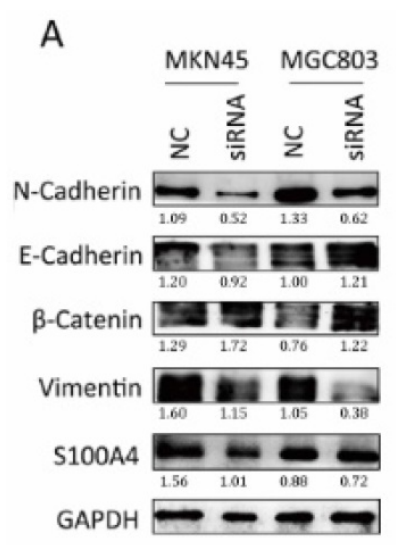

B

C
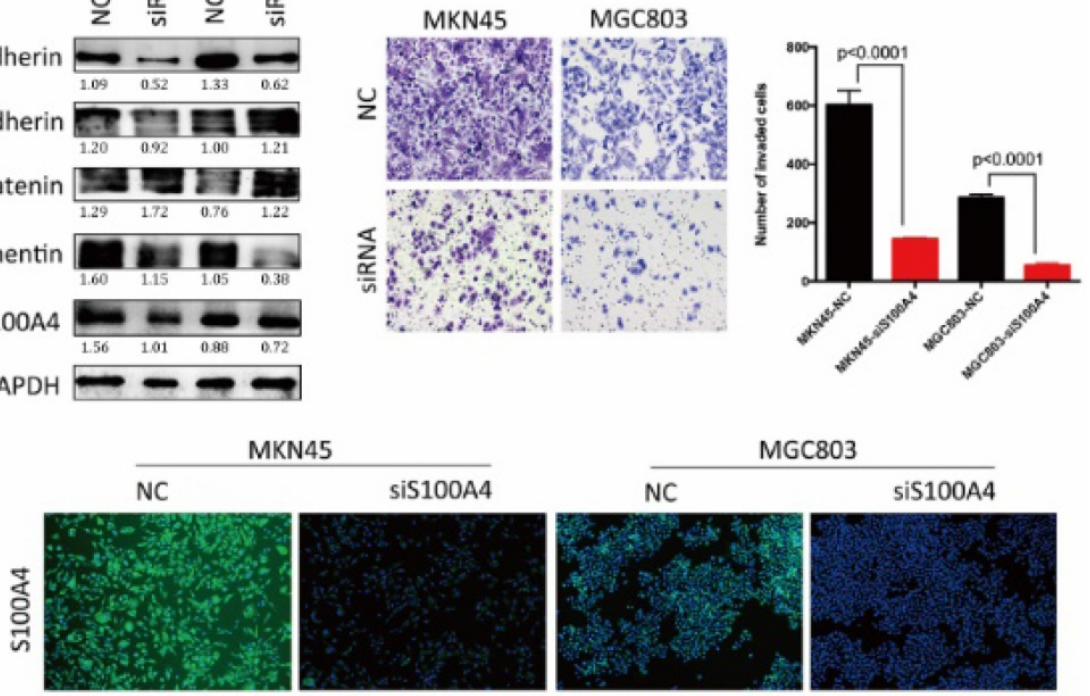

MGC803
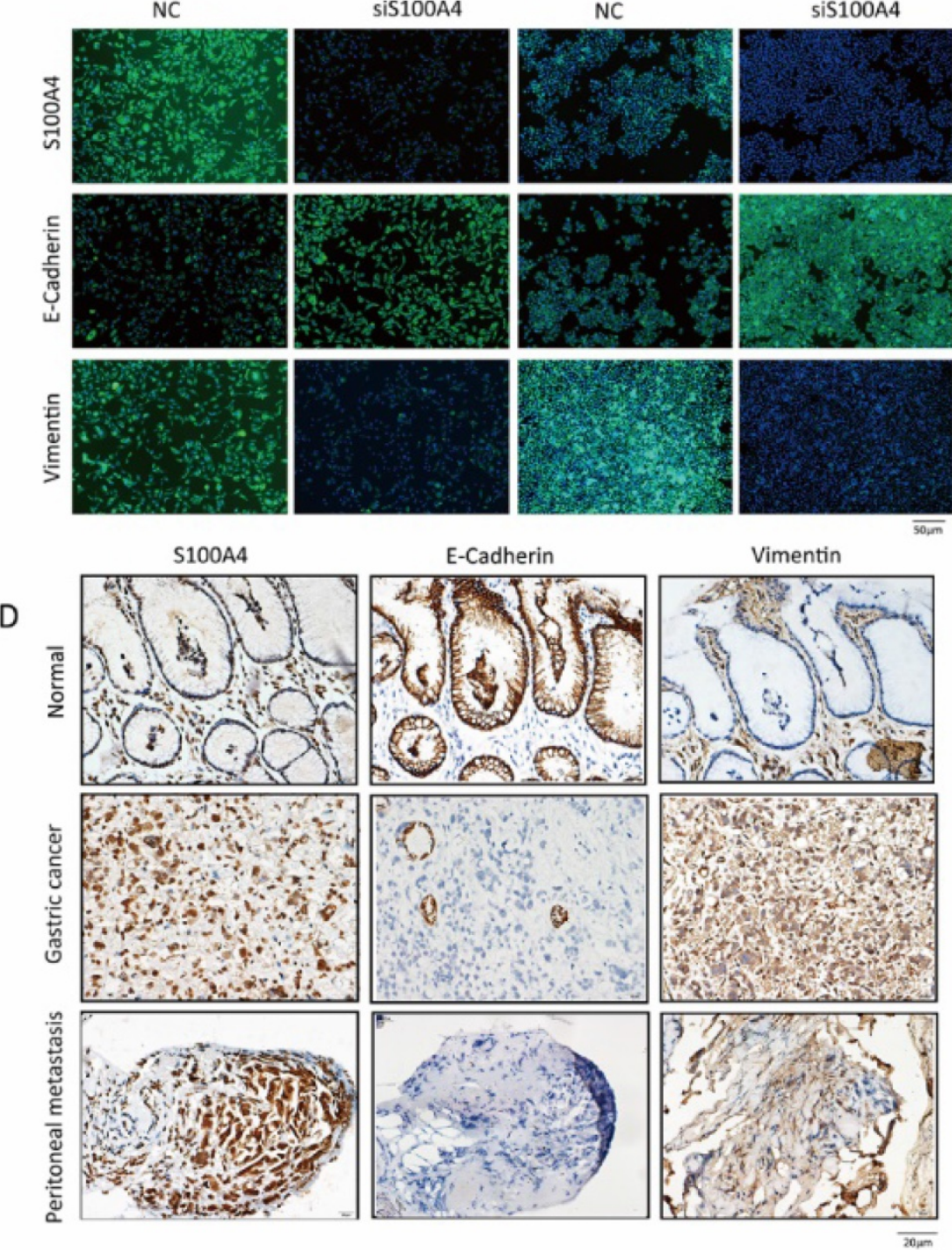

Figure 2. S100A4 was correlated with expression of EMT markers in gastric cancer tissue. Legends: A. Western blotting analysis of S100A4, Vimentin, $\beta$-Catenin, E-Cadherin and N-Cadherin in MKN45 and MGC803 cells. B. representative microscopic fields and cell counting of Transwell assay for MKN45 and MGC803 cells with interfered S100A4. C. subcellular localization of S100A4, Vimentin and E-Cadherin in MKN45 and MGC803 cells were assessed through immunofluorescence staining. D. IHC staining detected the expression of S100A4, E-Cadherin and Vimentin in gastric cancer peritoneal metastasis foci. 
A

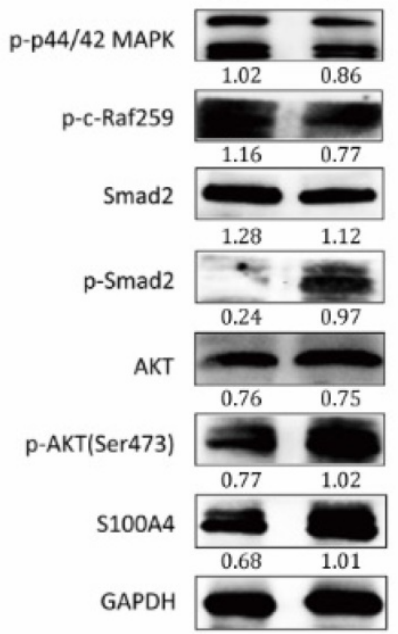

C

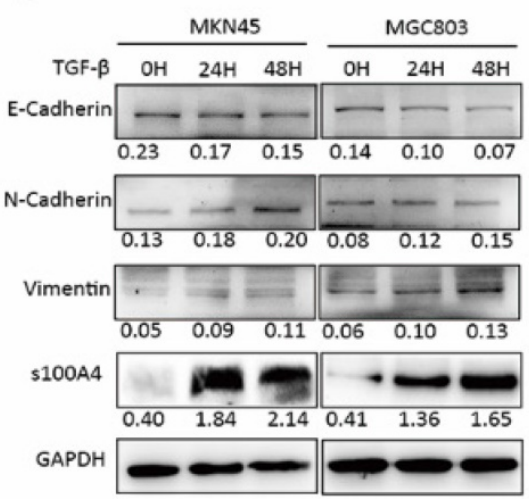

E

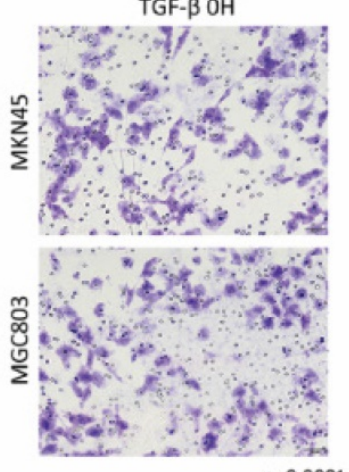
$\mathrm{p}<0.0001$

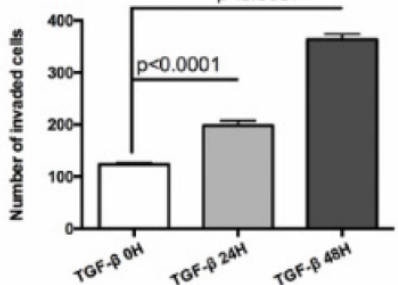

B

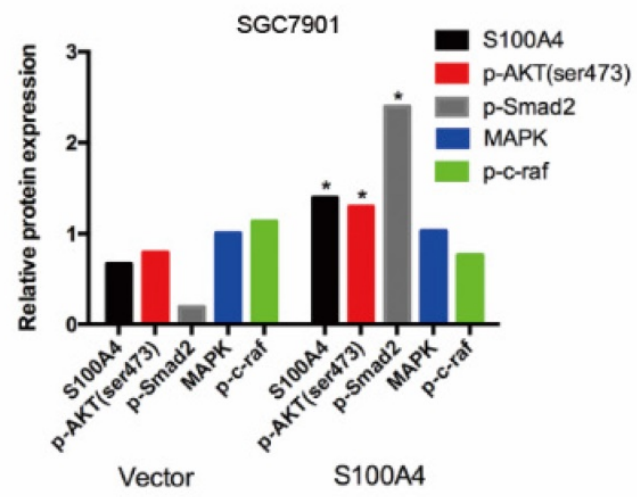

D

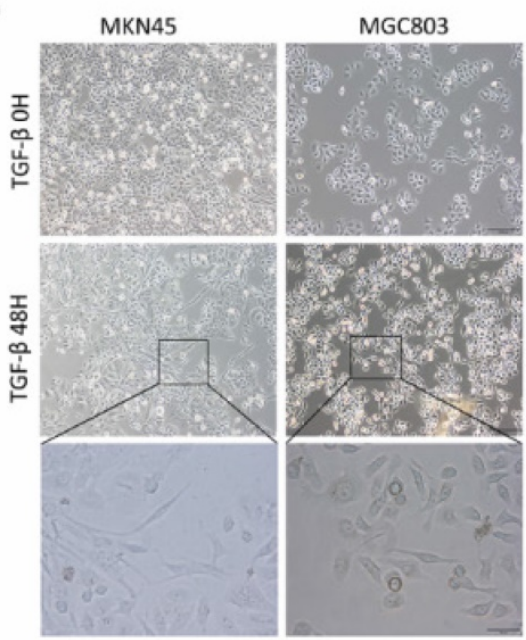

TGF- $\beta$ 48H
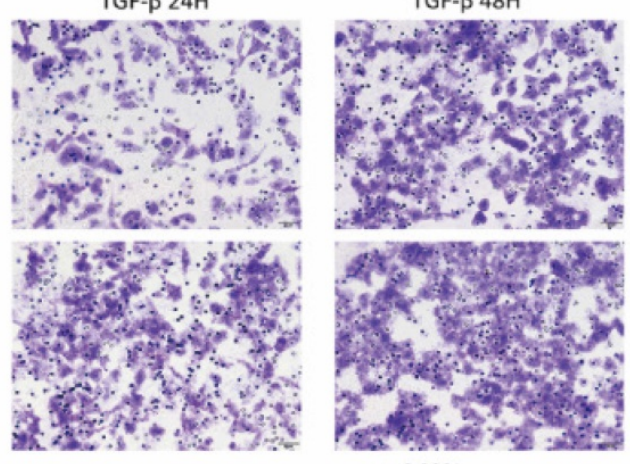

$p<0.0001$

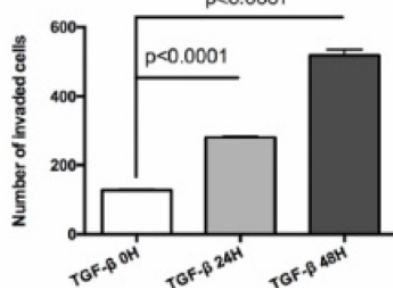

Figure 3. S100A4 regulated pivotal signaling transduction pathways. A. Western blotting for S100A4, MAPK, P44/42, P-AKT, Smad2, P-Smad2 in vector- or S100A4-transduced cells. B. the immunosignal was quantified using densitometric scanning software, and relative protein abundance was determined by normalization with internal control GAPDH. C. Western blot analysis was used to detect the expression of S100A4, E-Cadherin, N-cadherin and Vimentin in response to treatment of $10 \mathrm{ng} / \mathrm{ml}$ TGF- $\beta$ for $0,24,48$ hours. D. The morphologic change of gastric cancer cells in response to the treatment with TGF- $\beta$ or control for 48 hours was observed under an inverted microscope. E. The representative microscopic fields and statistical results of Transwell assay for MKN45 and MGC803 cells treated with TGF- $\beta$ v. 
A

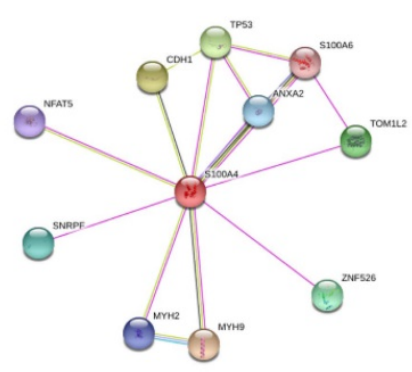

C

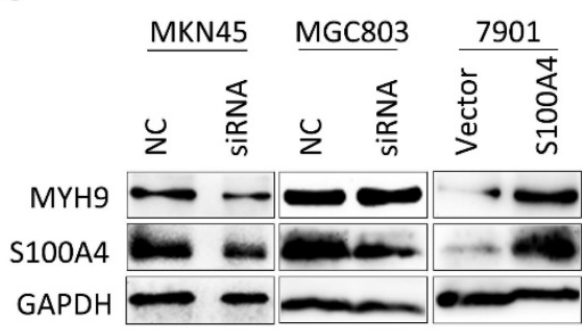

E

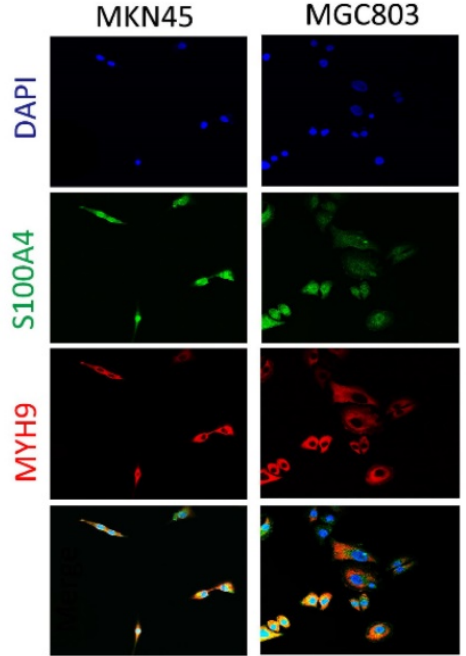

B

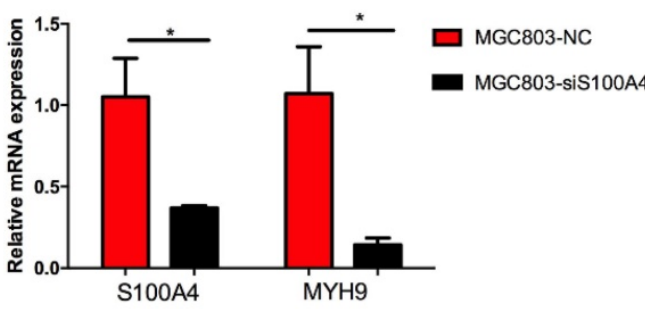

D

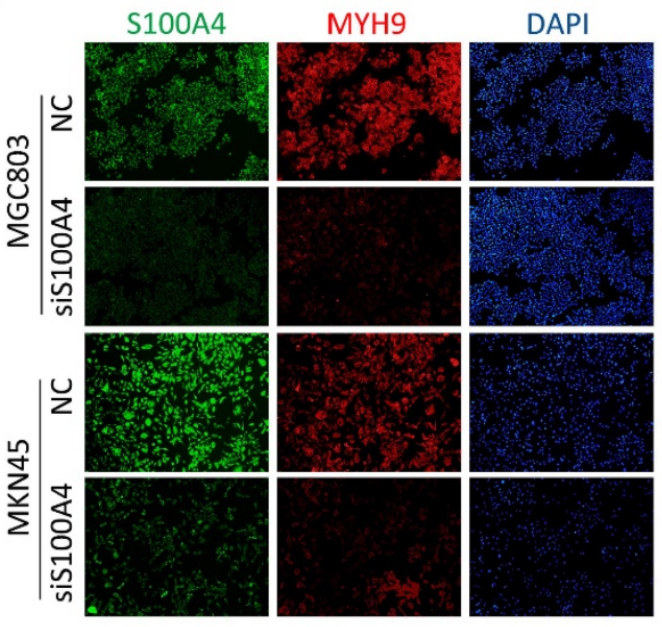

$$
\mathrm{F}
$$
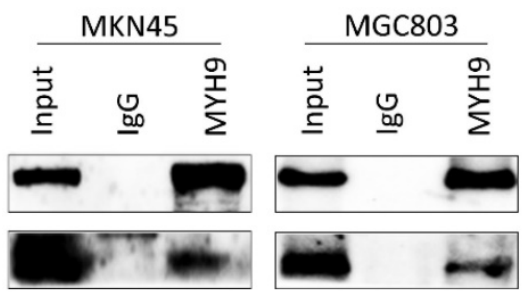

G

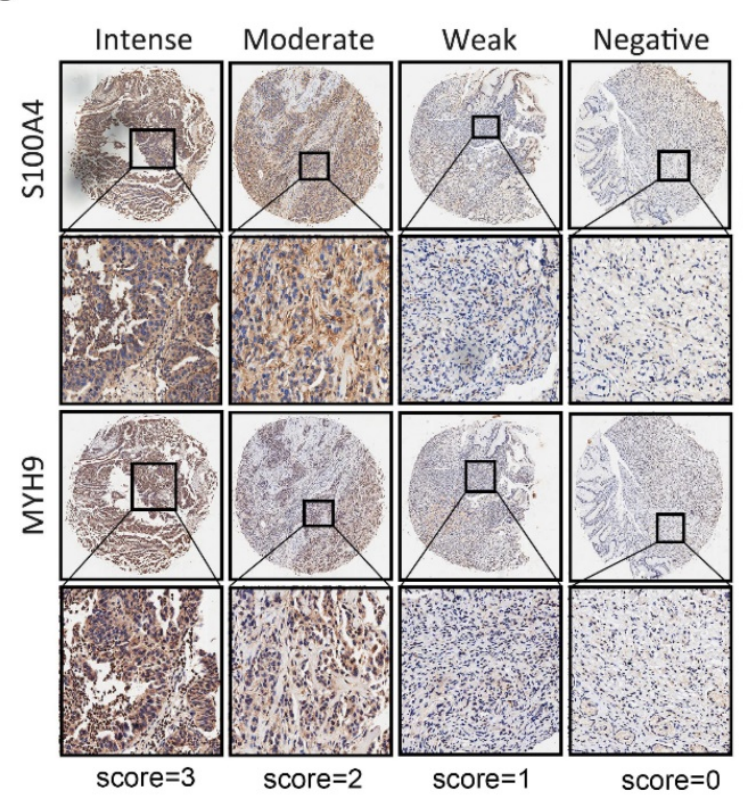

$\mathrm{H}$

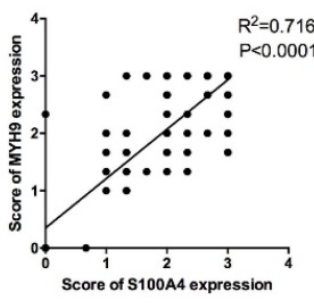

$\mathrm{J}$

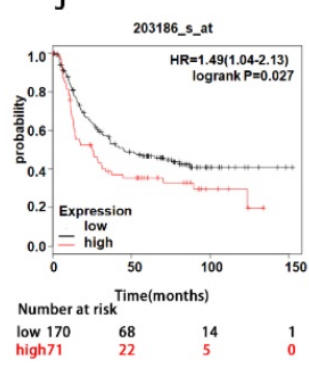

I

Figure 4. S100A4 was correlated with expression of MYH9 in gastric cancer tissue. Legends: A. String.com database indicate the downstream pivotal gene of S100A4. B. real-time PCR was performed to detect the expression of MYH9 and S100A4 mRNA in indicated cells. C-D. Western blot and immunofluorescence staining was performed to detect the expression of $\mathrm{MYH9}$ and S100A4 protein in indicated cells. E-F. The subcellular localization and interaction of MYH9 and S100A4 in indicated cells was assessed by immunofluorescence and coimmunoprecipitation respectively. G. TMA analysis of S100A4 and MYH9 in primary human gastric cancer tissues and normal adjacent tissues at $\times 100$ and $\times 200$ magnification. $H$. Regression analysis of S100A4 and MYH9 expression in primary gastric cancers $(n=90)$. Linear regression analysis was performed based on the expression score of S100A4 and MYH9 from the TMA. I-J-K. Kaplan-Meier survival analysis of S100A4 expression in patients with gastric cancer (log-rank test). The Kaplan-Meier analyses (log rank test) are shown for (I)all patients in the cohort and $(J)$ diffuse type or (K)stage IV GC patients using KM plotter, from public microarray data in GEO and TCGA database. 
A

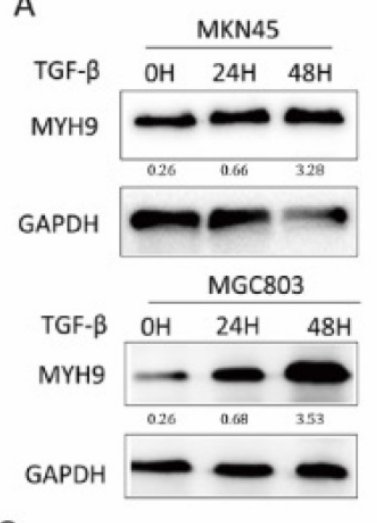

B

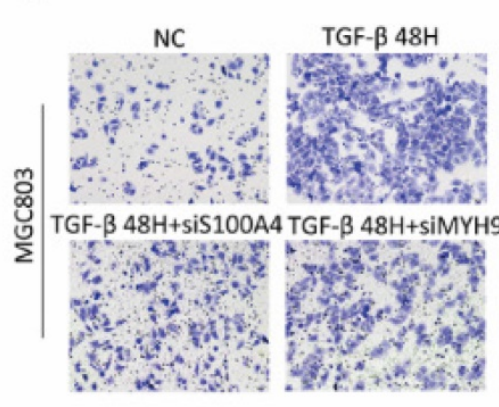

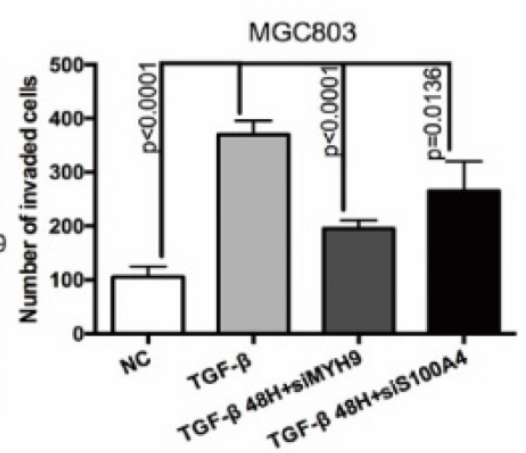

C

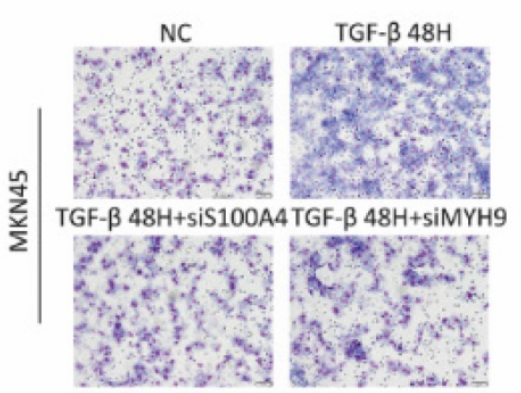

D

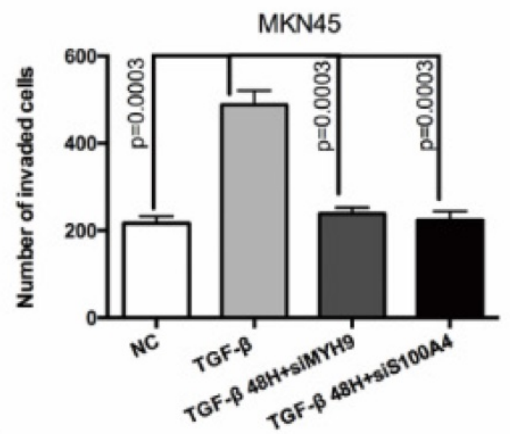

$E$
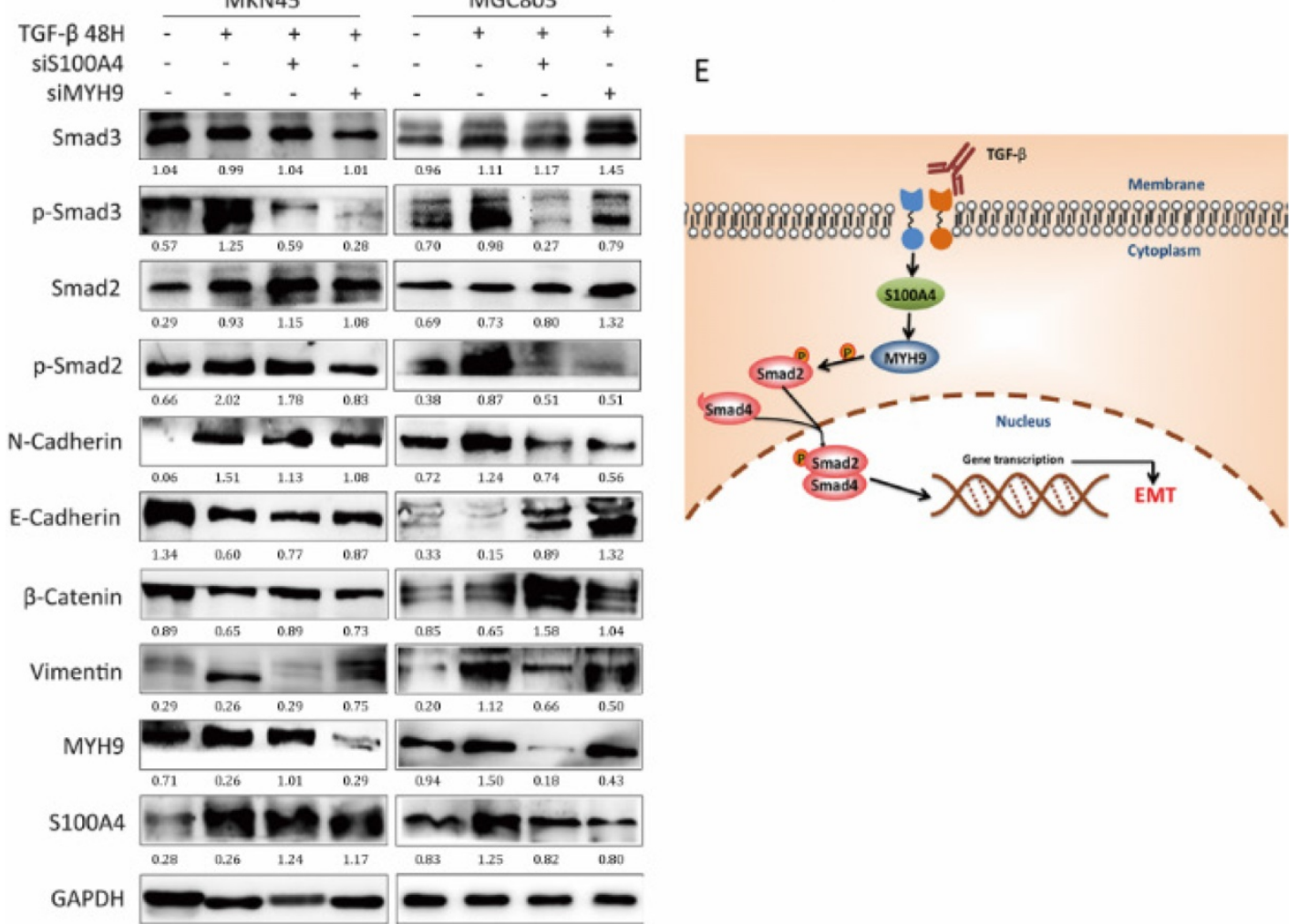

Figure 5. S100A4 and MYH9 are critical for TGF- $\beta$ mediated EMT process. Legends: A. Western blot of MYH9 in indicated cells in response to the treatment with $10 \mathrm{ng} / \mathrm{ml} \mathrm{TGF}-\beta$ for $0,24,48$ hours. B and C. The representative fields and statistic data of Transwell assay for MKN45 and MGC803. D. The Western blot of S100A4, MYH9, Vimentin, E-Cadherin, p44/42, MAPK, p-AKT473, AKT, Smad2, p-Smad2 in the indicated cells that were coinfected with 10ng/mL TGF- $\beta$ and S100A4 or MYH9 siRNA. E. a hypothetical model illustrating that S100A4 contributed to TGF- $\beta$-induced EMT by regulating MYH9.

\section{Discussion}

Recent researchers have identified S100A4 as a tumor metastasis-associated protein in many cancer, such as breast cancer[24], ovarian cancer[25], colorectal cancer[26], lung cancer[27],et.al. In our previous studies, S100A4 was identified as a protein that was associated with development and tumor 
progression of colorectal cancer [20]. Overexpression of S100A4 was found in metastatic gastric cancer and closely correlated with poor overall survival. The results suggested that S100A4 is a promising target in clinical therapy of patients with advanced gastric cancer. Nevertheless, the molecular mechanisms underlying S100A4 in peritoneal metastasis of gastric cancer are still unclear.

EMT played a critical role in cancer progression and metastasis. Numerous studies have illustrated that EMT is a common molecular mechanism in gastric cancer peritoneal metastasis. More and more evidences suggested that achievement of an EMT phenotype is associated with increased capability of invasion and metastasis in gastric cancer cells[28]. Up-regulation of mesenchymal markers and down-regulation of epithelial markers are the major molecular alteration during EMT. Taking this into consideration, we hypothesized that S100A4 is involved in EMT-mediated gastric cancer peritoneal metastasis. In this study, S100A4 expression was closely related with EMT markers in clinical gastric cancer samples. In vitro study has shown that the introduction of S100A4 induced changed expression levels of EMT associated markers accompanied with enhanced invasive ability of gastric cancer cell. Our results suggested that S100A4 probably participated in EMT-regulated progression in gastric cancer. We sought for molecular mechanism to shed light on biologic process leading to peritoneal metastasis in gastric cancer based on previous study. Then we used proteomic strategy to comprehensively explain S100A4-induced EMT processes.

Searching in STRING.com dataset, we revealed that one of the candidates downstream molecular of S100A4 was MYH9, which was positively regulated by S100A4. MYH9 is a gene encoding nonmuscle myosin IIA (NMIIA), belongs to the myosin II subfamily of actin-based molecular motors that includes skeletal, cardiac, smooth muscle, and no muscle myosin[29, 30]. Several studies have indicated that MYH9 played a key role in invasion and metastasis of cancer[31, 32].However, a study based on a direct in vivo siRNA strategy showed that MYH9 siRNA and MYH9 knockout significantly triggered formation of invasive squamous cell carcinoma. MYH9 is diminished in human SCCs and predict poor survival, suggesting it might act as a potential tumor suppressor[33]. Although further studies need to be carried out, our data provide new evidence that MYH9 might serve as a critical protein tend to interact with S100A4 to drive peritoneal metastasis in gastric cancer. In gastric cancer cells, exogenous overexpression of S100A4 remarkably enhanced the expression of MYH9 both in mRNA and protein level.
On the contrary, low expression of S100A4 obviously suppressed the expression of MYH9. The reinforcement of migration ability of gastric cancer cells caused by upregulated S100A4 was compromised when expression of MYH9 was suppressed. We believe that MYH9 was critical for S100A4 induced enhancement of invasive ability of gastric cancer.

Our study reveal that S100A4 can activate Smad signaling pathway by regulating phosphorylation level proteins in that pathway, which were recognized as pivotal link of cancer progression. TGF- $\beta$ induce EMT process through Smad and the complementary non-Smad pathway, containing MAPK, PI3K/AKT and p-c-raf259. In our study, both S100A4 and MYH9 were closely related with the TGF- $\beta /$ Smad pathway, a classical EMT mode. TGF- $\beta$ stimulation induced obvious changes of EMT-like phenotype and promoted migration ability of gastric cancer cells. We also observed a simultaneous increase of the expression of S100A4 and MYH9 in a time-dependent manner responding to TGF- $\beta$ stimulation. Moreover, S100A4 and MYH9 were indispensable for increasing the cell invasiveness of gastric cancer cells induced by TGF- $\beta$ stimulation. We propose that both of S100A4 and MYH9 are significant downstream effectors of the TGF- $\beta$ signaling pathway and TGF- $\beta$ activation produce invasive effects by the two proteins in gastric cancer cells.

Our study revealed that S100A4 could activate Smad signaling pathway by regulating phosphorylation level proteins in that pathway. TGF- $\beta$ induce EMT process through Smad and the complementary non-Smad pathway, containing AKT. In our study, both S100A4 and MYH9 were closely related with the TGF- $\beta /$ Smad pathway, a classical EMT mode. TGF- $\beta$ stimulation induced obvious changes of EMT-like phenotype and promoted migration ability of gastric cancer cells. We also observed a simultaneous increase in the expression of S100A4 and MYH9 in a time-dependent manner responding to TGF- $\beta$ stimulation. Moreover, S100A4 and MYH9 were required for increasing the cell invasiveness of gastric cancer cells induced by TGF- $\beta$ stimulation. We proposed that both of S100A4 and MYH9 were significant downstream effectors of the TGF- $\beta$ signaling pathway.

Overexpression of S100A4 was frequently found in gastric cancer tissues, especially in peritoneal metastatic tissues. In this study, S100A4 contribute to TGF- $\beta$-mediated EMT via Smad and signaling pathway. The phosphorylation protein interacted with various transcription factors and transcriptional coactivators or corepressors and regulated the transcription of target genes to promoted migration 
and invasiveness of gastric cancer cells. In conclusion, S100A4 contribute to progression of gastric cancer by playing a critical role in the TGF- $\beta$-mediated EMT process. Thus, S100A4 is a central molecular regulator during progression and metastasis of gastric cancer. Moreover, S100A4 may be employed to design specific targeted drugs for managing the patients with peritoneal metastasis of gastric cancer.

\section{Supplementary Material}

Supplementary tables.

http://www.jcancer.org/v09p3839s1.xlsx

\section{Acknowledgements}

The authors thank Liang Zhao (Department of Pathology, Nanfang Hospital, Southern Medical University, Guangzhou, Guangdong, China) for his valuable guidance on this research.

\section{Funding}

This work was supported by the grants from the State's Key Project of Research and Development Plan (2017YFC0108300, 2017YFC0108301), National Natural Science Foundation of China (81672446), Guangdong Provincial Science and Technology Key Project (2014A020215014), Research Fund of Public Welfare in the Health Industry, the National Health and Family Planning Commission of China (201402015), the Southern Medical University Clinical Research Start-Up Project (LC2016ZD003), Guangzhou Science and Technology Project (201400000004$5)$ and the Key Clinical Specialty Discipline Construction Program ([2011]170), President Foundation of Nanfang Hospital, Southern Medical University (2017Z012), the Special Funds for the Cultivation of Guangdong College Students' Scientific and Technological Innovation (pdjh2017b0107).

\section{Ethics approval and consent to participate}

This study was approved and conducted by Ethics Committee of Nanfang Hospital of Southern Medical University and all aspects of the study comply with the Declaration of Helsinki. Due to an anonymous analyzation only, informed consent to participate was not required.

\section{Availability of data and material}

All data generated or analyzed during this study are included in this published article while transcription levels and prognosis value of S100A4 in patients with gastric cancer are available in $\mathrm{KM}$ plotter (http:/ /www.kmplot.com) public analyzation platform updated biannually.

\section{Authors' contributions}

Guoxin $\mathrm{Li}$ and Hao Liu designed the experiments and revised the manuscript; Fengping Li, Jiaolong Shi and Zhijun $\mathrm{Xu}$ performed the experiments and wrote the manuscript; Xingxing Yao carried out the statistical analyzation; Tingyu Mou, Jiang Yu provided research materials.

\section{Competing Interests}

The authors have declared that no competing interest exists.

\section{References}

1. McLean $\mathrm{MH}$, El-Omar EM. Genetics of gastric cancer. Nature reviews Gastroenterology \& hepatology. 2014; 11: 664-74.

2. Torre LA, Bray F, Siegel RL, Ferlay J, Lortet-Tieulent J, Jemal A. Global cancer statistics, 2012. CA: a cancer journal for clinicians. 2015; 65: 87-108.

3. Hu Y, Huang C, Sun Y, Su X, Cao H, Hu J, et al. Morbidity and Mortality of Laparoscopic Versus Open D2 Distal Gastrectomy for Advanced Gastric Cancer: A Randomized Controlled Trial. J Clin Oncol. 2016.

4. Liu H, Li F, Zhu Y, Li T, Huang H, Lin T, et al. Whole-exome sequencing to identify somatic mutations in peritoneal metastatic gastric adenocarcinoma: A preliminary study. Oncotarget. 2016; 7: 43894-906.

5. Zhang J, Huang JY, Chen YN, Yuan F, Zhang H, Yan FH, et al. Whole genome and transcriptome sequencing of matched primary and peritoneal metastatic gastric carcinoma. Scientific reports. 2015; 5: 13750

6. Huang B, Sun Z, Wang Z, Lu C, Xing C, Zhao B, et al. Factors associated with peritoneal metastasis in non-serosa-invasive gastric cancer: a retrospective study of a prospectively-collected database. BMC cancer. 2013; 13: 57.

7. Kerkar SP, Kemp CD, Duffy A, Kammula US, Schrump DS, Kwong KF, et al. The GYMSSA trial: a prospective randomized trial comparing gastrectomy, metastasectomy plus systemic therapy versus systemic therapy alone. Trials. 2009; $10: 121$.

8. Kodera Y, Yamamura Y, Shimizu Y, Torii A, Hirai T, Yasui K, et al. Peritoneal washing cytology: prognostic value of positive findings in patients with gastric carcinoma undergoing a potentially curative resection. Journal of surgical oncology. 1999; 72: 60-4; discussion 4-5.

9. Yonemura $\mathrm{Y}$, Bandou E, Kinoshita K, Kawamura T, Takahashi S, Endou Y, et al. Effective therapy for peritoneal dissemination in gastric cancer. Surgical oncology clinics of North America. 2003; 12: 635-48.

10. Wu SM, Lin WY, Shen CC, Pan HC, Keh-Bin W, Chen YC, et al. Melatonin set out to ER stress signaling thwarts epithelial mesenchymal transition and peritoneal dissemination via calpain-mediated C/EBPbeta and NFkappaB cleavage. Journal of pineal research. 2016; 60: 142-54.

11. Roviello F, Marrelli D, de Manzoni G, Morgagni P, Di Leo A, Saragoni L, et al. Prospective study of peritoneal recurrence after curative surgery for gastric cancer. The British journal of surgery. 2003; 90: 1113-9.

12. Shah MA. Update on metastatic gastric and esophageal cancers. J Clin Oncol. 2015; 33: 1760-9.

13. Inoue K, Nakane $Y$, Michiura T, Nakai K, Sato M, Okumura S, et al. Trends in long-term survival following surgery for gastric cancer: a single institution experience. Oncology reports. 2004; 11: 459-64.

14. Qi XL, Zhang L, Lu XJ. New Insights into the Epithelial-to-Mesenchymal Transition in Cancer. Trends Pharmacol Sci. 2016; 37: 246-8.

15. Kalluri R, Neilson EG. Epithelial-mesenchymal transition and its implications for fibrosis. The Journal of clinical investigation. 2003; 112: 1776-84.

16. Iwatsuki M, Mimori K, Yokobori T, Ishi H, Beppu T, Nakamori S, et al. Epithelial-mesenchymal transition in cancer development and its clinical sionificance. Cancer science. 2010; 101: 293-9.

17. Garrett SC, Varney KM, Weber DJ, Bresnick AR. S100A4, a mediator of metastasis. J Biol Chem. 2006; 281: 677-80.

18. Barraclough $\mathrm{R}$, Dawson KJ, Rudland PS Control of protein synthesis in cuboidal rat mammary epithelial cells in culture. Changes in gene expression accompany the formation of elongated cells. European journal of biochemistry. 1982; 129: 335-41.

19. Wang H, Shi J, Luo Y, Liao Q, Niu Y, Zhang F, et al. LIM and SH3 protein 1 induces TGFbeta-mediated epithelial-mesenchymal transition in human colorectal cancer by regulating S100A4 expression. Clinical cancer research : an official journal of the American Association for Cancer Research. 2014; 20: 5835-47.

20. Ebralidze A, Tulchinsky E, Grigorian M, Afanasyeva A, Senin V, Revazova E, et al. Isolation and characterization of a gene specifically expressed in different metastatic cells and whose deduced gene product has a high degree of homology to a Ca2+-binding protein family. Genes \& development. 1989; 3: 1086-93. 
21. Shen ZY, Deng HJ, Fang Y, Zhu XJ, Ye GT, Yan L, et al. Identification of the interplay between SOX9 and S100P in the metastasis and invasion of colon carcinoma. Oncotarget. 2015; 6: 20672-84.

22. Zhao L, Wang H, Liu C, Liu Y, Wang X, Wang S, et al. Promotion of colorectal cancer growth and metastasis by the LIM and SH3 domain protein 1. Gut. 2010; 59: 1226-35.

23. Szasz AM, Lanczky A, Nagy A, Forster S, Hark K, Green JE, et al. Cross-validation of survival associated biomarkers in gastric cancer using transcriptomic data of 1,065 patients. Oncotarget. 2016; 7: 49322-33.

24. Pedersen KB, Nesland JM, Fodstad O, Maelandsmo GM. Expression of S100A4, E-cadherin, alpha- and beta-catenin in breast cancer biopsies. British journal of cancer. 2002; 87: 1281-6.

25. Kikuchi N, Horiuchi A, Osada R, Imai T, Wang CJ, Chen XJ, et al. Nuclear expression of S100A4 is associated with aggressive behavior of epithelial ovarian carcinoma: An important autocrine/paracrine factor in tumor progression. Cancer science. 2006; 97: 1061-9.

26. Atlasi Y, Noori R, Marolin I, Franken P, Brandao J, Biermann K, et al. The role of S100a4 (Mts1) in Apc- and Smad4-driven tumour onset and progression. European journal of cancer. 2016; 68: 114-24.

27. Kimura K, Endo Y, Yonemura Y, Heizmann CW, Schafer BW, Watanabe Y, et al. Clinical significance of S100A4 and E-cadherin-related adhesion molecules in non-small cell lung cancer. International journal of oncology. 2000; 16: 1125-31.

28. Huang L, Wu RL, Xu AM. Epithelial-mesenchymal transition in gastric cancer. American journal of translational research. 2015; 7: 2141-58.

29. Simons M, Wang M, McBride OW, Kawamoto S, Yamakawa K, Gdula D, et al. Human nonmuscle myosin heavy chains are encoded by two genes located on different chromosomes. Circulation research. 1991; 69: 530-9.

30. Toothaker LE, Gonzalez DA, Tung N, Lemons RS, Le Beau MM, Arnaout MA, et al. Cellular myosin heavy chain in human leukocytes: isolation of 5' cDNA clones, characterization of the protein, chromosomal localization, and upregulation during myeloid differentiation. Blood. 1991; 78: 1826-33.

31. Betapudi V, Licate LS, Egelhoff TT. Distinct roles of nonmuscle myosin II isoforms in the regulation of MDA-MB-231 breast cancer cell spreading and migration. Cancer research. 2006; 66: 4725-33.

32. Derycke L, Stove C, Vercoutter-Edouart AS, De Wever O, Dolle L, Colpaert N, et al. The role of non-muscle myosin IIA in aggregation and invasion of human MCF-7 breast cancer cells. The International journal of developmental biology. 2011; 55: 835-40.

33. Schramek D, Sendoel A, Segal JP, Beronja S, Heller E, Oristian D, et al. Direct in Vivo RNAi Screen Unveils Myosin IIa as a Tumor Suppressor of Squamous Cell Carcinomas. Science. 2014; 343: 309-13. 